\title{
The Spin-Spin Interaction and the New Concept of Photon
}

\author{
Quang Quy Ho \\ NEWTECHPRO, Vietnam Academy of Science \& Technology, Hanoi, Vietnam. \\ Email: hoquangquy@gmail.com, hoquy1253@yahoo.com
}

Received August 28 ${ }^{\text {th }}, 2012$; revised September 26 ${ }^{\text {th }}$, 2012; accepted October $6^{\text {th }}, 2012$

\begin{abstract}
After researching carefully the well known M. Planck's law of the black-body radiation, the quantum theory of field and the Einstein's postulates about interaction of the photon with the atoms, there are a lot of unclear questions about photon and its interaction with atom. From all the above questions, there are three main following questions: Why does the energy of a light mode include zero-point energy? Where does the first photon come from in universe? and What is the first fact as a reason of absorption, emission in the photon-atom interaction? To find out the acceptable answers, here we propose postulates about the zero-photon and then about the new concepts of photon. Using them, we have tried to describe the basic characters of the photon and explain the photon-atom interaction in other way: the spin-spin interaction. Our results showed out the different picture of mechanism of the photon-atom interaction, the existence of the zero-photon energy, the absorption as well as the emission rule and its probabilities.
\end{abstract}

Keywords: Zero-Point of Energy; Photon-Atom Interaction; Big Bang; Spin; Universe Wave

\section{Introduction}

Q1. Where is the zero-point energy from? If the explanation of "the presence of the zero-point energy can, however, be manifested in subtle ways when matter is exposed to static field" [1,2] is accepted, that means the matter transferred its energy $((1 / 2) \hbar \omega)$ to photon and consequence, each photon in a mode received this energy from matter, so the energy of a mode containing $n$ photons will be $E_{L}=n(1+1 / 2) \hbar \omega_{p}$, but not $E_{L}=(n+1 / 2) \hbar \omega_{p}$ as well known, till now.

Q2. Why a photon has rest mass equal zero [3-5], it is a material particle or energy-transfer unit?

Q3. If "the zero-point energy plays a crucial role in the process of spontaneous emission from an atom" [6], why the energy of photon of this process is $\hbar \omega_{p}$, but not, $(1 / 2) \hbar \omega_{p}$, consequently, why the frequency of photon is $\omega_{p}$ [7-10], but not $(1 / 2) \omega_{p}$ ?

Q4. Why a photon has spin $\pm \hbar$ [7-10], but not $\pm \hbar / 2$ as of the electron in atom?

Q5. Where do the first photons come from in the universe?

Q6. What is the way that the photon interacts with an electron? What is the condition for the electron in excited level forced to emit or to absorb photon? What does mean stimulation?

\section{Proposed Postulates}

Initially, we consider that the material world consisted of the vacuum and atoms, which is an ion surrounded by electrons [6], whose limit energy was one of two values $\pm e k \hbar / 2 \mu c$ relating to its spin value $\pm \hbar / 2$. If there was not any interaction from outside, electron (or atom) kept its inherent spin state (or orbital state) and consequence, no photon would be generated.

Postulate 1. After Big Bang, the energy storage in the universe is proposed to be expressed as follows

$$
m_{B H} c_{0}^{2}=E_{o b j}+E_{u v}=\sum_{i=1}^{N_{o}} \frac{m_{i} v_{i}^{2}}{2}+\sum_{j=1}^{N_{W}} n_{j} \hbar \omega_{z p, j}
$$

where, $m_{B H}$ is the mass of Black Hole, $c_{0}$ is the Einstein constant; $E_{o b j}$ is the total energy of all $N_{o}$ objects (element particles, galaxies, planets, ...), whose mass is $m_{i}$ and velocity is $v_{i} ; E_{u w}$ is the total energy of all $N_{W}$ universe waves, whose frequency is $\omega_{z p, j}$ and the number of the called "zero-point" of $j$-wave is $n_{j}$. The energy of "zero-point” generated from the Big Bang is defined as the energy of vacuum field, which can be independently quantized to energy unit $\hbar \omega_{z p}$ (from here it is called as "zero-photon") with spin $S_{z p}= \pm \hbar / 2$, mass $m_{z p}=0$ and velocity $c_{0}$.

Postulate 2. A photon is the fancy quantum teeter of two identical "zero-photons" coupling one with other by a fancy shoulder pole with a fancy fulcrum. It propagates in the direction perpendicularly to fancy shoulder pole (see Figure 1) by a zigzag around its fancy fulcrum as shown in Figure 2.

Postulate 3. The photon is linearly polarized when its spin lays on the plane containing propagating axis and 


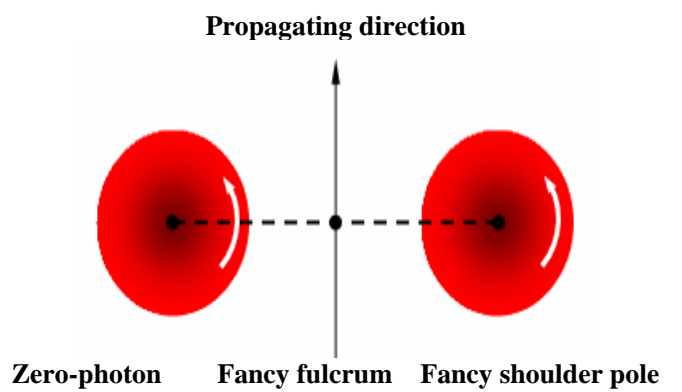

Figure 1. Photon as a fancy quantum teeter of two zerophoton.

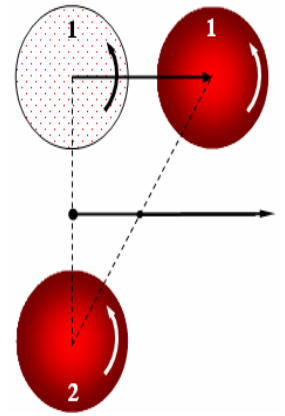

(a)

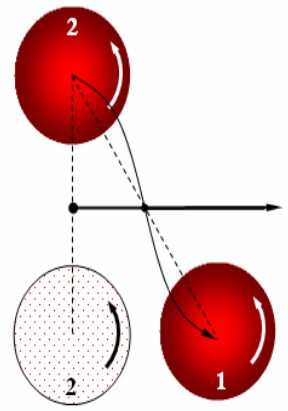

(b)
Figure 2. Oscillation of the photon (a) Linear polarized photon: advancing-zigzag in the plane containing propagating axis ana spin; (b) Left-circulaly polarized photon: rotating-zigzag around propagating axis.

fancy shoulder. The photon is circularly polarized when its spin lays in the plane containing its spin and perpendicular to propagating axis (see Figure 2).

Postulate 4. Energy emission and absorption processes of the atom appear when its inherent spin-state is changed by spin-spin interaction with zero-photon.

\section{Answer to above Questions}

With proposed postulates, we can clearly answer all the above questions.

Answer 1. This question is answered by Postulate No. 1. From here, the zero-photon is considered as the initial jostle to swing photon.

Answer 2. Because of the mass of zero-photons is zero, so the rest mass of photon

$$
m_{\text {rest, } p h}=2 m_{z p}=0 \text {. }
$$

From postulates 1 and 2, a photon is considered as energy-transfer unit only, but not material.

Answer 3. Frequency of photon is defined as the oscillation frequency of the fancy fulcrum (Figure 3). After jostling of the vacuum field, two zero-photons oscillate with a period

$$
T_{z p}=\frac{1}{\omega_{z p}}
$$

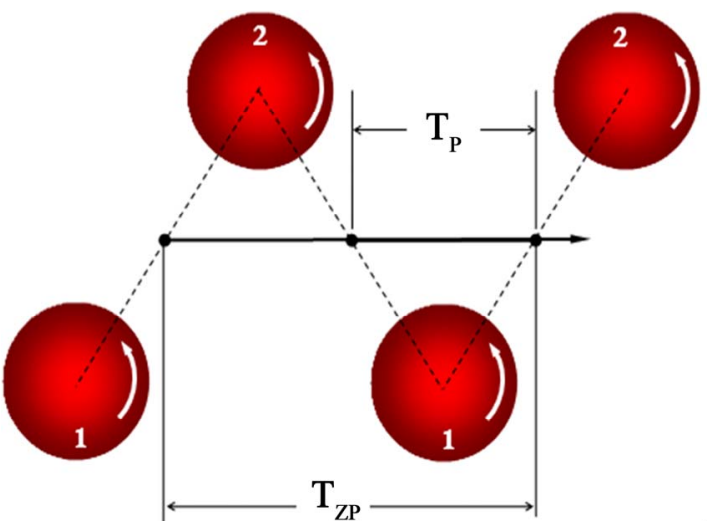

(a)

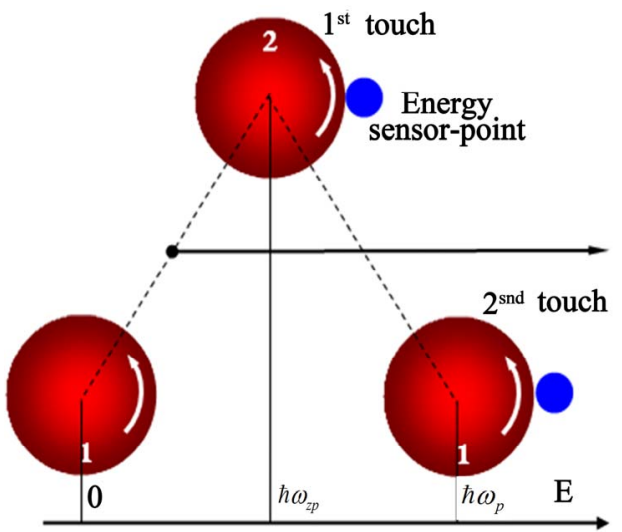

(b)

Figure 3. Frequency description (a) and energy description (b) of one photon.

meanwhile, the fancy fulcrum (or photon) oscillates with the period

$$
T_{p}=\frac{T_{z p}}{2} .
$$

From (2) and (3), we have frequency of photon

$$
\omega_{p}=\frac{1}{T_{p}}=\frac{2}{T_{z p}}=2 \omega_{z p}
$$

Equation (4) can be confirmed by the energy description of a photon. Assume that in oscillating process two zero-photons one by one touch the sensitive point of the detector, so when a photon completed one touching, the sensitive point of detector received energy:

$$
E_{p}=2 E_{z p}=2 \hbar \omega_{z p}=\hbar \omega_{p}
$$

It means $\omega_{z p}=\omega_{p} / 2$.

Answer 4. Consider two zero-photons have spin of $+\hbar / 2$ (or $-\hbar / 2)$ one by one touch the sensitive point of the spin detector (Figure 4). At each touching a spin of $-\hbar / 2$ (or $+\hbar / 2$ ) is registered. So, after completely touching of one photon the sensitive point of the spin detector registered a spin of 


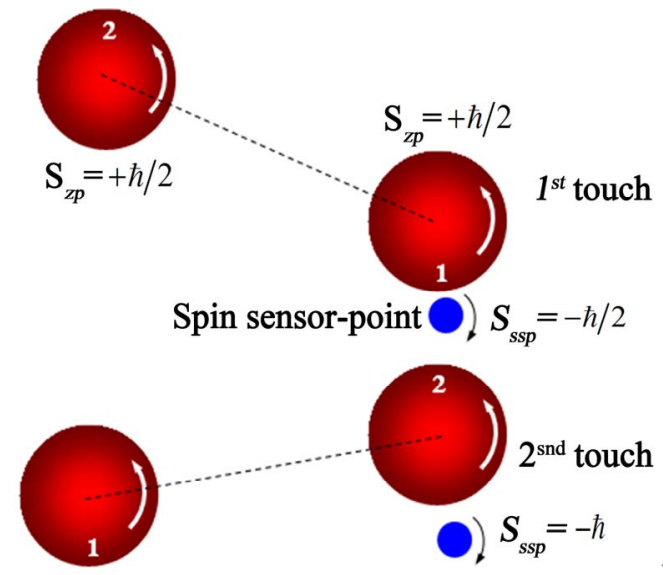

Figure 4. Touching-sensitive point process of the photon gives its spin $+\hbar$.

$$
\left\{\begin{array}{l}
S_{p}=2 S_{z p}=2(-\hbar / 2)=-\hbar \\
\text { or } \\
S_{p}=2 S_{z p}=2(+\hbar / 2)=+\hbar
\end{array}\right.
$$

and, consequence, the spin of the photon is $\pm \hbar$.

Answer 5. The spontaneous emission is the source of first photons in the universe. The spontaneous emission can be explained from suddenly losing and then changing of the inherent spin-state of electron by the zero-photon. As well known an electron has its inherent spin of $\pm \hbar / 2$. Consider after the Big Bang the electron excited in the called "excited level" correlating to an energy of $\left(E_{g}+2 \hbar \omega_{z p}\right)_{e}$, where $E_{g}$ is the energy of the called "ground level". Suddenly, this electron lost its inherent spin-state by considered coherent zero-photon with $E_{z p}=\hbar \omega_{z p}$, and $S_{z p}= \pm \hbar / 2$ propagating in the vacuum. There are four variants of interaction depending on the commutation of the spin signs of the electron and zerophoton.

Variant 1. $S_{e}=+\hbar / 2, \quad S_{z p}=+\hbar / 2$. The changing process of the electron spin could be described in the Figure 5. The electron suddenly is braked by one coming zero-photon of the vacuum field and then its spin momently equals zero $\left(S_{z p}=0\right)$. Because of its inertia, at that moment a new zero-photon with spin $+\hbar / 2$ is separated from the electron (see first step in Figure 5). After the first step, the electron transfers its energy to the new zero-photon and falls down to the vitual energy level. Since its inertia, from this level the electron changes its spin from $S_{e}=0$ to $S_{e}=-\hbar / 2$ to separate one more zero-photon with spin of $S_{z p}=+\hbar / 2$, and continuously falls down to the ground level (second step in the Figure 5). In the end of this process, one photon consisted of two new zero-photons with spin of $S_{p}=+\hbar$ is generated.

After the first step, the coming zero-photon went away, while new zero-photon continuously obliges electron to
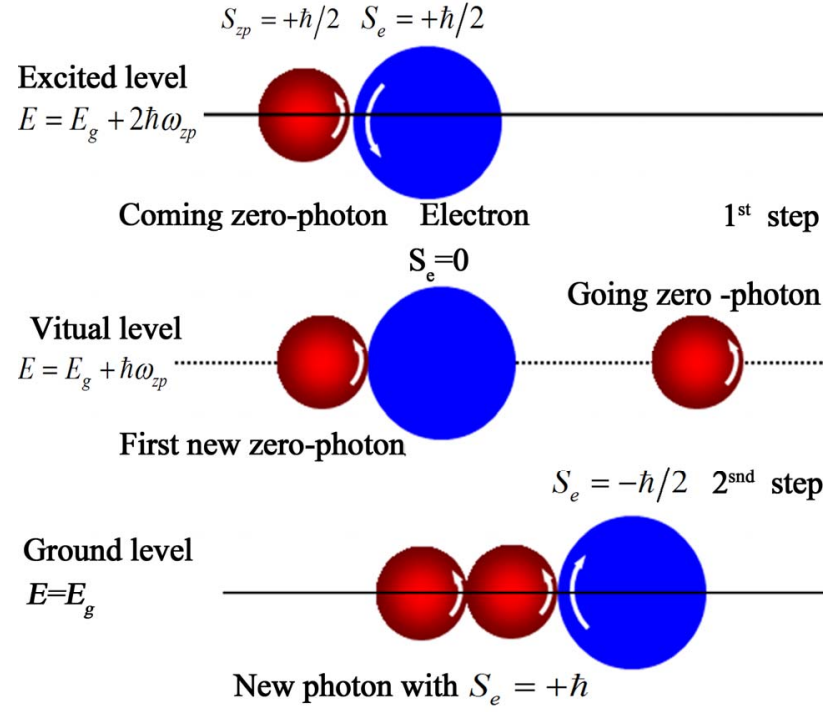

Figure 5. Process of spontaneous emission for variant 1.

change. After the second step, the changing process of the spin in the electron-zero-photon interaction can be written as follows:

$$
\begin{aligned}
\left(+\frac{1}{2} \hbar\right)_{e} & \Rightarrow(0 \hbar)_{e}+\left(+\frac{1}{2} \hbar\right)_{z p} \\
& \Rightarrow\left(-\frac{1}{2} \hbar\right)_{e}+2\left(+\frac{1}{2} \hbar\right)_{z p} \\
& \Rightarrow\left(-\frac{1}{2} \hbar\right)_{e}+(+\hbar)_{z p}
\end{aligned}
$$

From (A7) results that

$$
S_{e}^{\prime}=-\frac{1}{2}=+\frac{1}{2}-1=S_{e}-1
$$

Since the spin inherence of electron, comparing (8) with Pauli rule it is shown that after changing its spin, the electron must be in different energy state, which is corresponding to the difference of spin, $\Delta S=-1$, that means the electron should transit down to lower level and transfer its energy to photon. The new photon appears.

Variant 2. $S_{e}=-\hbar / 2, S_{z p}=-\hbar / 2$.The changing process of the spin moment of electron is similar to that in Figure 5, excepted in the ground level the spin of electron is $S_{e}^{\prime}=+\hbar / 2$ and of photon $S_{p}=-\hbar$.

Variant 3. $S_{e}=+\hbar / 2, S_{z p}=-\hbar / 2$ and

Variant 4. $S_{e}=-\hbar / 2, S_{z p}=+\hbar / 2$.

The changing process of the moment for both variants is described in Figure 6. From this figure can see that the spin of electron is unchanged by the attraction the coming zero-photon. In the first step, the electron swallows the coming zero-photon and is excited in the vitual level with energy of $E=E_{g}+3 \hbar \omega_{z p}$. This phenomenon is similar to that the big trip attracted the small boat in its 


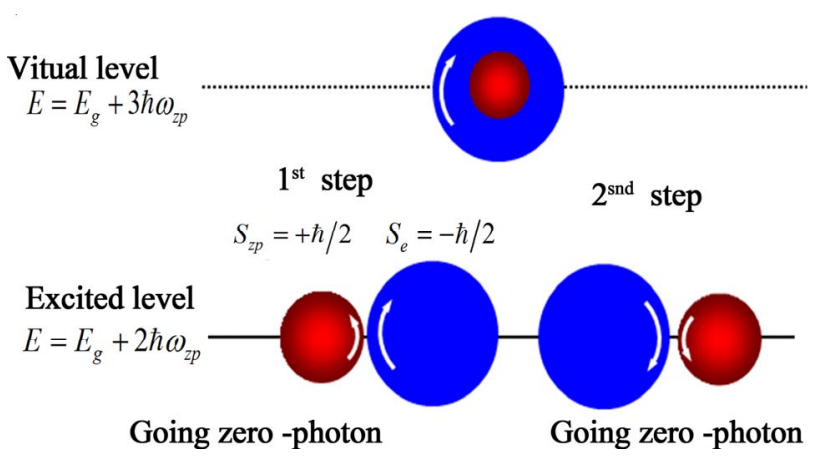

Figure 6. Process of the zero-photon electron interaction for variant 3 and variant 4 .

high-speed trace. Since that any interaction with other zero-photon goes in the vitual level, the electron speedily relaxes down to the initial level and the zero-photon goes out.

We conclude that the spontaneous emission to generate a photon may be appeared with the folowing conditions: 1) The first jostle of the zero-photon of vacuum field is the necessary reason; 2 ) The excited electron and coming "zero-photon" having the same sign of spin is the sufficient condition; 3) The resonant energy, i.e. $\Delta E=2 \hbar \omega_{z p}$ is the sufficient condition.

Answer 6. Stimulated absorption can be explained by coherently attracting coming photon of electron in the ground level (see Figure 7). The first zero-photon of the coming photon is coherently attracted by the electron. After swallowing the zero-photo, the electron transits upto the vitual level (see first step in Figure 7). Because of the teeter's configuration of the photon, in this level, the second zero-photon of the coming photon is coherently attracted by electron also (see second step in Figure 7), and then photon is swallowed up by the electron, which transites upto excited resonant level.

Depending on the spin sign of electron and coming photon, there are four spin-spin interaction variants of the electron and coming photon, but attracting process should be appeared (or an electron will absorved the photon ) for two variants, when electron and photon have the opposite sign of spin. The spin relation of the electron is given as follows:

$$
\left(+\frac{1}{2}\right)_{e} \Rightarrow\left(+\frac{1}{2}\right)_{e}
$$

From (11) results that

$$
S_{e}^{\prime}=+\frac{1}{2}=+\frac{1}{2}=S_{e}
$$

Relation (10) shows that electron will transit from lower level to higher level due to the difference of $\Delta S=0$ and the absorb a photon.

Stimulated emission can be explained by changing

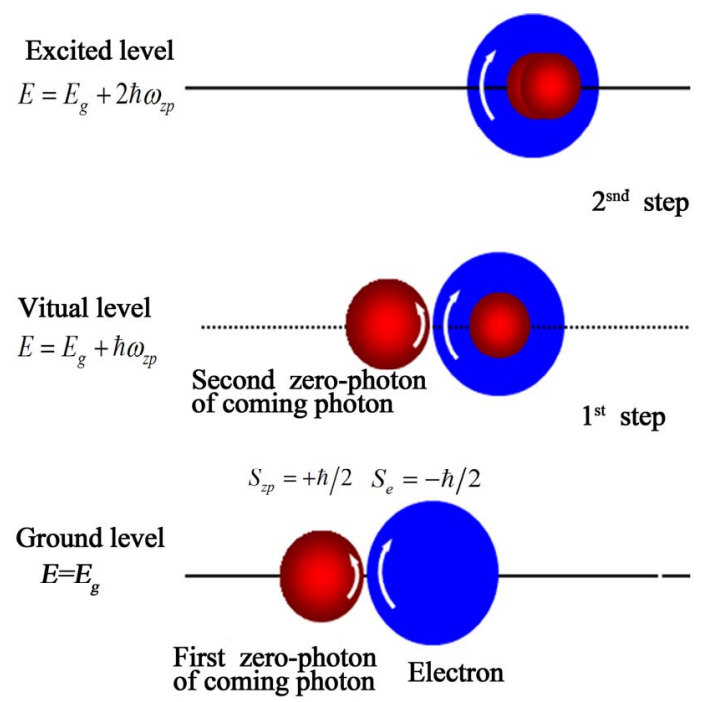

Figure 7. Process of stimulated absorption.

inherent spin-state of electron in excited level after touching with coming photon. There are four variants of the interaction depending on the commutation of spin signs of the electron and photon. As shown in the case of spontaneous emission, two variants $S_{e}=+\hbar / 2$, $S_{p}=+\hbar$ and $S_{e}=-\hbar / 2, \quad S_{p}=-\hbar$ will be accepted. The stimulated emission is described in Figure 8.

The electron suddenly is braked by the first zero-photon of coming photon and then its spin momently equal zero. Because of its inertia, at that moment a new zerophoton with spin $+\hbar / 2$ is separated from electron, which falls downto the vitual level (see first step in Figure 8). After the first step, the second zero-photon continuously obliges electron to change its spin from 0 to $-\hbar / 2$, and the second new zero-photon with $+\hbar / 2$ is separated (see second step in Figure 8). After second step, the changing process of the spin of the electron can be written as follows:

$$
\begin{aligned}
\left(+\frac{1}{2}\right)_{e} & \Rightarrow(0)_{e}+\left(+\frac{1}{2}\right)_{z p} \\
& \Rightarrow\left(-\frac{1}{2}\right)_{e}+2\left(+\frac{1}{2}\right)_{z p} \\
& \Rightarrow\left(-\frac{1}{2}\right)_{e}+(+1)_{p}
\end{aligned}
$$

From (13) results that

$$
S_{e}^{\prime}=-\frac{1}{2}=+\frac{1}{2}-1=S_{e}-1
$$

From (12) and what shown in Figure 8, we can see that the excited electron transits from the higher energy level to lower energy level and generates a new photon with spin as well as that of the coming photon after changing its spin, $\Delta S=-1$. Moreover, as shown in 


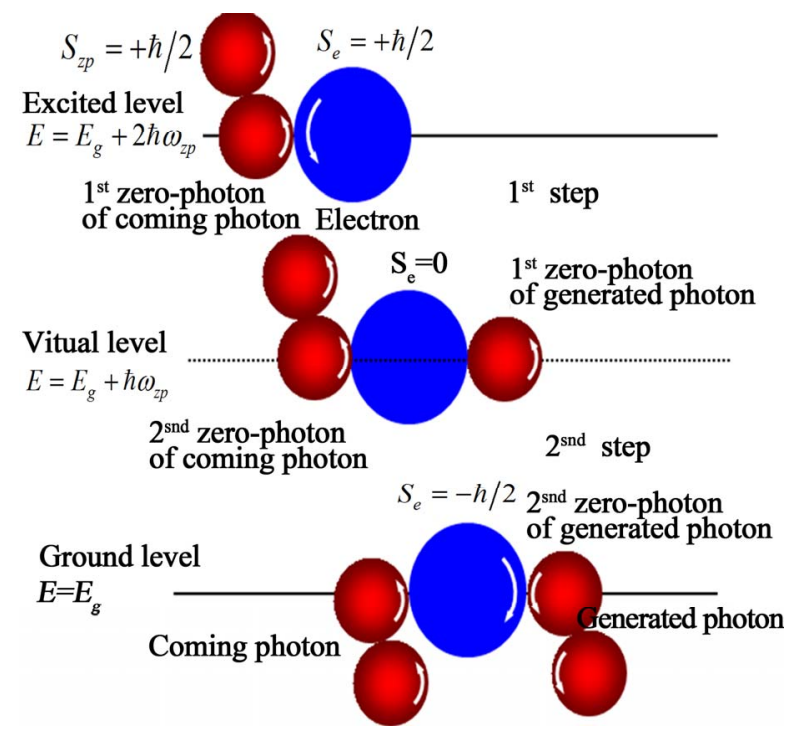

Figure 8. Process of stimulated emission.

Figure 8, the new photon is separated from electron only when the obliging of the coming photon finishes, i.e. the new photon always follows the coming photon. This description can more clearly explain the coherent property of photons after the stimulated emission.

\section{Conclusions}

From all shown above, we can give some points in conclusion.

The vacuum field is an existence with its energy unit, which called "zero-photon" in the universe and its oscillation is a main-basic reason to generate photon.

The photon is a fancy-quantum teeter of two same zero-photon.

A zero-photon propagating in the vacuum can brake any electron in its path to generate photons by its spin not by its energy. So the energy of light field consisting of $n$ photons in the vacuum is a sum of photon energy and zero-photon energy of vacuum field, i.e.,

$$
E_{L}=(2 n+1) \hbar \omega_{z p}=\left(n+\frac{1}{2}\right) \hbar \omega_{p} .
$$

The spin-state of electron $( \pm \hbar / 2)$, “zero-photon” $( \pm \hbar / 2)$ and photon $( \pm \hbar)$ is inherent as a natural behavior of them.

The main mechanism of the electron-photon interaction is the spin-spin interaction, which is seen as the beginning fact of the photon-matter interaction.
The spontaneous emission will appeare when the electron lost its inherent spin-state by the zero-photon of vacuum field.

The stimulated emission will appeare when the electron lost its spin by braking (stimulating) of the opposite spin photon.

The absorption photon will appeare when the electron and photon are themselves attracted by their spin with same sign.

Depending on the spin state of all electron in the atom (molecule) and the acceptable variant of spin sign of electron and photon, the spontaneous emission, stimulated absorption and stimulation emission have their determined spin-spin interaction probability.

The questions, postulates and answers presented above are considered as the stupid proposals. I hope they give us the opportunity to discuss about some related phenomena.

\section{REFERENCES}

[1] B. E. A. Saleh and M. C. Teich, "Fundamentals of Photonics,” John Wiley \& Sons, INC, New York, 1998.

[2] John Manchak, "Arguments Concerning Photon concepts," 2006. file://P:00LD\old_aporia_sitelvolumelvol132〉photons.ht m

[3] M. Austern, "What is the mass of a photon?" 2008. http://math.ucr.edu/home/baez/physics/ParticleAndNucle ar/photon_mass.html

[4] A. Zajonc, R. Loudon, et al., "Optics \& Photonics,” OPN Trend, Vol. 14, No. 10, 2003, S1-S34.

[5] J. P. Siepmann, "What is a photon?” 2004. http://d1002391.mydomainwebhost.com/JOT/Editorials/ Vol-6/e6-3.htm

[6] P. A. M. Dirac, “The Principle of Quantum Mechanics,” 4th Edition, Clarendon, Oxford, 1958.

[7] S. Weinberg, "The Quantum Theory of Fields," Cambridge University Press, Cambridge, 2000.

[8] A. Yariv, "Introduction to the Theory and Applications of Quantum Mechanics,” Willey, New York, 1982.

[9] H. Haken, “Light: Waves, Photons, Atoms,” North-Holland, Amsterdam, 1981.

[10] E. Wolf, "Einstein's Researches on the Nature of Light," Optics News, Vol. 5, No. 1, 1979, pp. 24-39. doi:10.1364/ON.5.1.000024 